\title{
ORIGINAL
}

\section{OPINIÓN DE LOS MÉDICOS DE ATENCIÓN PRIMARIA DE OURENSE SOBRE ALGUNOS ASPECTOS DE SU PRESCRIPCIÓN FARMACÉUTICA}

\author{
Gabriel J Díaz Grávalos (1), Gerardo Palmeiro Fernández (1), Eloína Núñez Masid (2) e \\ Inmaculada Casado Górriz (3)
}

(1) Centro de Salud Cea (Ourense)

(2) Dirección Gerencia de Atención Primaria (Ourense)

(3) Centro de Salud Allariz (Ourense)

\section{RESUMEN}

Fundamento: Conocer la opinión de los médicos de atención primaria de Ourense (España) con respecto a algunos aspectos de su prescripción como el conocimiento del precio de los fármacos, la prescripción inducida percibida, la relación con la industria farmacéutica y la opinión del médico sobre posibles medidas reductoras del gasto farmacéutico.

Métodos: En este estudio transversal descriptivo, se entrevistó mediante un cuestionario postal, previamente probado, a todos los médicos de atención primaria (MAP) de la provincia de Ourense (243). En él se recogían características demográficas de los facultativos, influencia del coste en la prescripción, estimación del precio de 15 fármacos de uso habitual y la opinión sobre diversos aspectos relacionados con la prescripción inducida, la industria farmacéutica y diversas estrategias para contener el gasto en farmacia. Para el análisis de los resultados se utilizaron pruebas de $\chi^{2}$, $t$ de Student y análisis de la varianza, así como el coeficiente de correlación de Spearman según cumpliera con un error $\alpha$ de 0,05 .

Resultados. El nivel de participación de los encuestados fue del 42,8\% (104). La duración media diaria de la visita de los delegados farmacéuticos fue de 13,6 minutos, siendo las novedades el punto de mayor interés. Casi un $27 \%$ admitía participar en ensayos clínicos patrocinados por la industria. El $23 \%$ consideraba el precio una prioridad al prescribir. La prescripción inducida suponía el 39,7 \% del total. Los facultativos eligieron mayoritariamente el copago para reducir el gasto farmacéutico. En la estimación del precio el porcentaje de error global fue de $45,7 \%$, subestimando los más caros y sobrestimando los más baratos.

Conclusiones. Existe un desconocimiento importante de precio de los fármacos entre los MAP. La mayor parte de los médicos no consideran que el precio del fármaco deba ser una prioridad al prescribir. Hay un porcentaje elevado de prescripción inducida percibida. Respecto al gasto farmacéutico, la mayor parte de los MAP considera las medidas económicas, en concreto el copago, como las más efectivas para su contención.

Palabras clave: Gasto farmacéutico. Atención primaria. Prescripción de medicamentos.

Correspondencia:

Gabriel J Díaz Grávalos

Feria 9

32130 Cea (Ourense)

Correo electrónico: gdgravalos@hotmail.com

\section{ABSTRACT}

Opinion of the Primary Care Physicians of Ourense with respect to certain Aspects of their Prescription of Medicines

Background: To learn the opinion of the primary care physicians of Ourense (Spain) with respect to certain aspects of their prescription of medicines, such as their awareness of the price of drugs, the induced prescribing perceived, their relationship with the pharmaceutical industry and their opinions concerning possible measures for reducing the expenditure on medicines.

Methods: In this transversal descriptive study, all of the primary care physicians in the province of Ourense (243) were surveyed by means of a previously-approved questionnaire sent to them by post. The questionnaire included demographic characteristics of the physicians, the influence of cost when prescribing medicines, their estimate of the price of 15 drugs routinely prescribed and their opinion on different aspects relating to induced prescription, the pharmaceutical industry and different strategies in order to curtail the expenditure on medicines. In order to analyze the results, the $\chi^{2}$ and Student's t tests and an analysis of the variance were used, together with the Spearman correlation coefficient with $\alpha=0.05$.

Results. The level of participation of the physicians surveyed was $42.8 \%$ (104). The average daily duration of visits by pharmaceutical company representatives was 13.6 minutes, with new specialities being the topic of greatest interest. Close to $27 \%$ admitted to having participated in clinical testing sponsored by the industry. $23 \%$ considered the price to be a priority when prescribing. Induced prescription accounted for $39.7 \%$ of the total. The majority of physicians chose co-payment as the means for reducing expenditure on medicines. In the estimate of prices, the overall percentage of error was $45.7 \%$, underestimating the more expensive medicines and overestimating the less expensive.

Conclusions. There is a significant lack of awareness of the price of drugs among the primary care physicians. Most of the physicians do not feel that the price of a drug should be a priority when prescribing medicines. There is a high percentage of induced prescription perceived. With regard to the expenditure on pharmaceuticals, the majority of those surveyed feel that financial measures, specifically co-payment, are the most effective in order to curtail rising costs.

Key Words: Medical care cost. Primary care. Prescription. Medicines. 


\section{INTRODUCCIÓN}

Una de las principales preocupaciones de las Administraciones Sanitarias es el gasto farmacéutico, no sólo en términos absolutos, sino también por su ritmo de incremento, notablemente superior al crecimiento general de los precios. Durante el año 2000, se gastó en el Sistema Nacional de Salud más de un billón de pesetas en farmacia (un $7,4 \%$ más respecto a 1999 , con un incremento del número de recetas del $4,9 \%$ y un gasto promedio por receta de 1874 pesetas) $)^{1}$.

Para explicar este gasto se han desarrollado diversas interpretaciones que implican a los prescriptores, a la industria farmacéutica (IF), a la Administración y a los ciudadanos, receptores y usuarios finales de los fármacos. A tenor de estos diversos condicionantes de la prescripción, intrínsecos y extrínse$\cos$ al médico ${ }^{2}$, que intentan explicar los motivos de una determinada elección, se han diseñado estrategias para intentar que el gasto disminuya o al menos no continúe aumentando con el ritmo actual.

Siempre se ha achacado a los facultativos un escaso interés respecto a cuestiones económicas, alegando que considerarían que deben prescribir aquello que en su opinión es más beneficioso para el paciente, independientemente del precio. Sin embargo, diversos estudios dibujan un panorama diferente, donde con frecuencia la prescripción no responde a un criterio exclusivamente clínico: formación académica, presión asistencial, entorno laboral y mercadotecnia industrial ${ }^{2-4}$.

En el proceso de adquisición de cualquier bien, y la prescripción de fármacos lo es, conocer el precio del producto es una condición indispensable para conseguir un resultado lo más eficiente posible. Sin embargo, existen serias dudas de que los médicos que prescriben conozcan con precisión aceptable el precio de los fármacos que indican ${ }^{5-8}$.
Los objetivos de este estudio han sido valorar el conocimiento que sobre los precios de los fármacos de uso habitual tienen los facultativos de Atención Primaria de la provincia de Ourense, la importancia que el precio les merece a la hora de prescribir, la influencia de la prescripción inducida desde el punto de vista del médico que la recibe y las medidas que a su juicio deberían aplicarse para reducir el gasto en farmacia.

\section{MATERIAL Y MÉTODOS}

Para la realización de este estudio descriptivo transversal, entre el mes de noviembre de 1999 y el mes de septiembre de 2000 se envió un cuestionario postal a todos los médicos de Atención Primaria (MAP) de la provincia de Ourense (243 individuos). El listado de los facultativos, así como las direcciones profesionales de los mismos, se obtuvieron a través de la Gerencia de Atención Primaria del Servicio Galego de Saúde.

El envío iba acompañado de una carta de presentación del estudio, firmada por la directora médica de Atención Primaria de la provincia, explicando las características del mismo y asegurando la absoluta confidencialidad de los datos. Cada cuestionario, aunque anónimo, llevaba una identificación numérica en su primera hoja para facilitar un segundo envío a los no respondedores. Una persona ajena al estudio, administrativo de la Gerencia, disponía de la relación numérica y nominal de cada encuestado. A ella se remitían los cuestionarios una vez cumplimentados, por correo interno del Servicio Galego de Saúde (SERGAS), cuando era factible, o mediante correo ordinario. No se facilitó sobre de respuesta. Tras contactar telefónicamente con los médicos que no contestaron en el plazo estipulado para solicitarles que lo hicieran, se les volvía a remitir un segundo cuestionario al cabo de un tiempo prudencial.

Este cuestionario, construido al efecto, había sido previamente probado mediante 
su administración a un grupo de 17 médicos de atención primaria de dos centros de salud, C.S. Cambre (A Coruña) y C.S. Aldebarán (Madrid), para detectar posibles fallos de construcción; quedó constituido definitivamente por 27 preguntas, 8 de tipo sociodemográfico, 8 sobre la relación con la industria farmacéutica, 5 sobre prescripción inducida percibida, 5 sobre el precio de los fármacos y otros aspectos de la prescripción y una sobre las medidas más adecuadas para reducir el gasto farmacéutico (anexo 1). La selección de los 15 fármacos sobre los que se indagaba el precio se basó en los listados de fármacos más prescritos en la provincia en los 3 meses anteriores.

Una vez recibidos y tabulados los resultados, se calculó la variable principal del estudio «Porcentaje de error de cada médico en la estimación del precio de cada producto farmacéutico» ( $\% \Delta \mathrm{MPF})$, prescrito o no, calculado según la fórmula $\left(100 \times \frac{\mathrm{E}}{\mathrm{R}}\right)$ donde $\mathrm{E}$ es el precio estimado por el facultativo y $\mathrm{R}$ el precio real en la fecha de la encuesta (un valor 100 indica coincidencia entre precio estimado y real). El tamaño muestral mínimo para la estimación de dicha variable, con una precisión del $10 \%$, un $\alpha$ de 0,05 y una desviación estándar de 34 (tomada de Oppenheim $^{5}$ ) dio un resultado de 51 individuos.

Otras variables calculadas fueron:

- Media de la diferencia (precio estimado-precio real) por producto farmacéutico.

- Media del \% $\%$ MPF por producto farmacéutico.

- Porcentaje global de error de estimación de los facultativos: se hallaron los valores promedio de las desviaciones (como valores absolutos de $\mid 100-$ $\% \Delta$.M.PF) de cada facultativo en todas las especialidades farmacéuticas prescritas por él, y luego se calculó la me- dia de dichos porcentajes promedio personales.

- Porcentaje global de error de estimación por producto farmacéutico: de forma análoga, se hallaron para cada producto farmacéutico los valores promedio de las desviaciones (como valores absolutos de $\mid 100-\% \Delta$.M.PF $\mid)$ de todas las estimaciones emitidas (se prescribiera el fármaco o no), y posteriormente se calculó la media de dichos promedios.

Se realizó un análisis comparativo entre las características sociodemográficas (edad, sexo, años de permanencia en el trabajo, situación laboral, modelo integrado o no) de los respondedores y los no respondedores, éstas obtenidas a través de la Gerencia de Atención Primaria de Ourense.

El análisis de los datos se realizó con la ayuda de los programas estadísticos SPSS 9.0 y EPIDAT 2.0.

Las características básicas de la muestra fueron analizadas con métodos univariantes, representando las variables cualitativas mediante estimación de proporciones y las cuantitativas mediante media y desviación estándar [ $\overline{\mathrm{x}}(\mathrm{DE})]$, ambas con sus correspondientes intervalos de confianza del $95 \%$ $\left(\mathrm{IC}_{95 \%}\right)$.

La relación entre variables cualitativas se analizó con la prueba de $\chi^{2}$; entre variables cualitativas y cuantitativas a través de la comparación de medias mediante una prueba t de Student o análisis de la varianza; y entre variables cuantitativas mediante el coeficiente de correlación de Spearman, previa comprobación del cumplimiento de las condiciones de aplicación de las diversas pruebas.

\section{RESULTADOS}

Participaron 104 médicos, lo que supone un porcentaje de respuesta del $42,8 \%$, con 
una media de edad de 42,5 (6,1) años, siendo un $42,3 \%$ (44) mujeres. Los principales resultados sociodemográficos, de los aspec- tos de la visita y de la relación con la industria farmacéutica quedan resumidos en la Tabla 1.

Tabla 1

Datos sociodemográficos y aspectos de la visita y de la relación con la industria farmacéutica más relevantes

\begin{tabular}{|c|c|c|}
\hline & $\%(n) o \bar{x} \pm D E$ según proceda & $I C_{95 \%}$ \\
\hline \multicolumn{3}{|l|}{ Aspectos sociodemográficos } \\
\hline Nivel de participación & $42,8 \%(104)$ & \\
\hline Por sexos & $42,3 \%(44)$ mujeres & \\
\hline Media de edad & $42,5 \pm 6,1$ años & {$[41,3 \sim 43,7]$} \\
\hline Antigüedad & $15,0 \pm 6,3$ años & {$[13,8 \sim 16,3]$} \\
\hline \multicolumn{3}{|l|}{ Situación laboral } \\
\hline Propietarios: & $39,4 \%(41)$ & {$[30,1 \sim 49,4]$} \\
\hline Interinos: & $56,7 \%(59)$ & {$[46,6 \sim 66,2]$} \\
\hline Sustitutos: & $3,9 \%(4)$ & {$[1,2 \sim 10,1]$} \\
\hline Modelo Integrado & $67,3 \%(70)$ & {$[57,3 \sim 75,9]$} \\
\hline \multicolumn{3}{|l|}{ Medio poblacional: } \\
\hline$<5.000$ habitantes: & $62,5 \%(65)$ & {$[52,4 \sim 71,6]$} \\
\hline 5.000-25.000 habitantes: & $21,1 \%(23)$ & {$[13,9 \sim 30,4]$} \\
\hline$>25.000$ habitantes: & $15,4 \%(16)$ & {$[9,3 \sim 24,1]$} \\
\hline Población atendida (en TIS) & $1277 \pm 514$ & [1162 1391] \\
\hline Pacientes/día: & $39,4 \pm 39,4$ & {$[36,3 \sim 42,6]$} \\
\hline \multicolumn{3}{|c|}{ Aspectos de la visita y de la relación con la industria farmacéutica } \\
\hline Delegados/día & $2,8(0,9)$ & {$[2,6 \sim 2,9]$} \\
\hline Duración de la visita (minutos) & $13,6(5,7)$ & {$[9,2 \sim 17,8]$} \\
\hline \multicolumn{3}{|l|}{ Interés en el contenido de la visita } \\
\hline Novedades & $51,9 \%(54)$ & {$[41,9 \sim 61,7]$} \\
\hline Aspectos farmacológicos & $44,2 \%(46)$ & {$[34,5 \sim 54,2]$} \\
\hline Precio & $1 \%(1)$ & {$[0,0 \sim 6,0]$} \\
\hline \multicolumn{3}{|l|}{ Actitud ante las novedades: espera... } \\
\hline información escrita & $59,6 \%(62)$ & {$[49,5 \sim 68,9]$} \\
\hline aceptación generalizada & $33,7 \%(35)$ & {$[24,9 \sim 43,7]$} \\
\hline opinión de colegas & $5,8 \%(6)$ & {$[2,3 \sim 12,6]$} \\
\hline Prescribe tan pronto se lo presentan & $1 \%(1)$ & {$[0,0 \sim 6,0]$} \\
\hline Lectura de estudios de la industria & $54 \%(56)$ & {$[43,9 \sim 63,7]$} \\
\hline Participación en estudios patrocinados & $26,9 \%(28)$ & {$[18,8 \sim 36,6]$} \\
\hline Acepta el patrocinio de actividades formativas & $83,7 \%(87)$ & {$[74,8 \sim 89,9]$} \\
\hline
\end{tabular}

TIS: tarjetas sanitarias individuales 
No se encontraron diferencias significativas en las características de los facultativos respondedores y no respondedores, salvo en el mayor porcentaje de respondedores que ejercían en municipios de menos de 5.000 habitantes.

La totalidad de los médicos que contestaron admitía recibir la visita de delegados farmacéuticos, con un promedio de 2,8 $(0,9)$ por día $\left(\mathrm{IC}_{95} \%[2,6 \sim 2,9]\right)$, siendo más frecuentes estas visitas conforme aumentaba el número de TIS del facultativo $(\rho=0,3$; $\mathrm{p}=0,003)$.

Un 26,9 \% (28) admitía participar en estudios patrocinados por la industria farmacéutica, y un 5,8\% no respondió a esta cuestión. Por otra parte, 87 médicos $(83,7 \%)$ creían adecuado el patrocinio por parte de los laboratorios farmacéuticos de cursos y otras actividades formativas.

El 76,0 \% (79) $\left(\mathrm{IC}_{95 \%}[66,4 \sim 83,5]\right)$ de los encuestados admitió fijarse en el precio al recetar, pero sólo el $23,1 \%$ (24) ( $\mathrm{IC}_{95 \%}$ $[15,6 \sim 32,5])$ consideraba el precio una prio- ridad a la hora de prescribir, sin diferencias entre sexo ni edad. El 8,7\% no contestó a esta pregunta.

La tabla 2 muestra los precios reales de las distintas especialidades farmacéuticas (ordenados de forma ascendente) en el momento de realizar la encuesta y las diferentes medias de las estimaciones efectuadas y de las desviaciones en las estimaciones en pesetas. En la tabla 3 se indican los porcentajes mínimo y máximo estimados para cada especialidad farmacéutica, ordenadas también de forma ascendente según su precio real, y su media (con $\mathrm{DE} \mathrm{e} \mathrm{IC}_{95} \%$ ). El porcentaje global de error en la estimación de los facultativos fue del 45,7\% $(27,9)$ $\left(\mathrm{IC}_{95 \%}[38,8 \sim 52,7]\right)$ con mínimo de $8,4 \%$ y máximo de $136 \%$. Si se consideran los diferentes fármacos, el porcentaje global de error de estimación por producto farmacéutico se puede estimar en más de un $37,3 \%$ $(35,6)\left(\mathrm{IC}_{95 \%}[19,3 \sim 55,3]\right)$, con extremos entre el 6,6\% (Norvas ${ }^{\circledR} 5,30$ comp) y el $153,7 \%$ (Meleril $\left.^{\circledR}, 10 \mathrm{ml}\right)$. En conjunto, se subestimaba el precio de los fármacos de mayor coste, mientras se sobreestimaba el

Tabla 2

Precios reales y promedios de las estimaciones efectuadas y de las diferencias entre lo estimado y lo real

\begin{tabular}{|c|c|c|c|c|}
\hline Presentación Farmacéutica & Precio Real * & $\begin{array}{l}\text { Media de la esti- } \\
\text { mación }\end{array}$ & $\begin{array}{c}\text { Media de la dife- } \\
\text { rencia } \\
\text { (estimado-real) }\end{array}$ & Desviación típica \\
\hline Termalgin 50020 comp & 239 & 386 & 147 & 220 \\
\hline Meleril $10 \mathrm{ml}$ & 244 & 619 & 375 & 672 \\
\hline Voltarén emulgel 60 gr & 603 & 825 & 222 & 262 \\
\hline Trankimazin 0,530 comp & 661 & 985 & 324 & 689 \\
\hline Ventolín inh & 681 & 943 & 263 & 635 \\
\hline Almax forte 1,5 gr $30 \mathrm{sob}$ & 795 & 1.136 & 341 & 768 \\
\hline Voltarén 5040 comp & 1.066 & 1.031 & -91 & 410 \\
\hline Diemil $180 \mathrm{ml}$ & 1.529 & 1.229 & -299 & 471 \\
\hline Monocid 1 g IM 1 vial & 1.822 & 1.465 & 356 & 638 \\
\hline Zantac 15028 comp & 2.861 & 2.298 & -562 & 662 \\
\hline Norvas 530 comp & 3.050 & 2.849 & -200 & 912 \\
\hline Renitec 2028 comp & 3.460 & 2.848 & -612 & 774 \\
\hline Pulmicort TBH 400100 dosis & 5.489 & 3.617 & -1.871 & 1.368 \\
\hline Zarator 1028 comp & 5.538 & 4.278 & -1.259 & 1.107 \\
\hline Seroxat 2028 comp & 5.768 & 4.162 & -1.605 & 1.371 \\
\hline
\end{tabular}

* Tomado de Catálogo de Especialidades Faramaceúticas 1999. (en ptas.) 
de los más económicos. El porcentaje de error en la estimación se asociaba de forma significativa con el número de pacientes en consulta $(\rho=0,28 ; p=0,03)$ y con el número de delegados de visita farmacéutica recibidos $(\rho=0,38 ; p=0,002)$.

Tabla 3

Porcentajes de error en las estimaciones *

\begin{tabular}{|c|c|c|c|c|c|}
\hline Presentación farmacéutica & Mínimo & Máximo & Media & $\begin{array}{l}\text { Desviación } \\
\text { típica }\end{array}$ & $I C_{95 \%}$ \\
\hline Termalgin 50020 comp & 83,7 & 627,6 & 161,7 & 92,3 & {$[138,6 \sim 184,8]$} \\
\hline Meleril $10 \mathrm{ml}$ & 81,9 & $2.049,2$ & 253,7 & 275,5 & {$[182,3 \sim 325,0]$} \\
\hline Voltarén emulgel $60 \mathrm{gr}$ & 16,6 & 298,5 & 136,9 & 43,5 & {$[125,8 \sim 148,0]$} \\
\hline Trankimazin 0,530 comp & 30,2 & 605,1 & 149 & 104,3 & {$[121,9 \sim 176,0]$} \\
\hline Ventolín inh & 29,3 & 513,9 & 138,6 & 93,3 & {$[114,6 \sim 162,6]$} \\
\hline Almax forte $1,5 \mathrm{gr} 30 \mathrm{sob}$ & 62,9 & 880,5 & 142,9 & 96,7 & {$[118,2 \sim 167,6]$} \\
\hline Voltarén 5040 comp & 35,6 & 267,1 & 91,8 & 36,5 & {$[82,5 \sim 101,1]$} \\
\hline Diemil $180 \mathrm{ml}$ & 19,6 & 170,1 & 80,4 & 30,8 & {$[72,2 \sim 88,5]$} \\
\hline Monocid $1 \mathrm{~g}$ IM 1 vial & 16,5 & 219,5 & 80,4 & 35,0 & {$[71,3 \sim 89,4]$} \\
\hline Zantac 15028 comp & 17,5 & 139,8 & 80,3 & 23,1 & {$[74,3 \sim 86,2]$} \\
\hline Norvas 530 comp & 26,2 & 163,9 & 93,4 & 29,9 & {$[85,6 \sim 101,1]$} \\
\hline Renitec 2028 comp & 20,2 & 138,7 & 82,3 & 22,3 & {$[76,5 \sim 88,1]$} \\
\hline Pulmicort TBH 400100 dosis & 12,9 & 109,3 & 65,9 & 24,9 & {$[59,5 \sim 72,2]$} \\
\hline Zarator 1028 comp & 14,4 & 108,3 & 77,2 & 20,0 & {$[72,0 \sim 82,3]$} \\
\hline Seroxat 2028 comp & 17,3 & 104 & 72,1 & 23,7 & {$[66,0 \sim 78,1]$} \\
\hline
\end{tabular}

* Un valor de 100 indica coincidencia entre precio estimado y real.

Más del $87 \%$ de la muestra refería tener mucha o bastante fidelidad a las especialidades que prescribía. Entre las razones aportadas para justificar la prescripción de una determinada marca comercial frente a otra destacan la antigüedad del nombre comercial en el mercado (30\%) y el precio (30\%), influyendo en menor medida la variedad de presentaciones. Sin embargo, no todos los fármacos se comportaban igual en este aspecto. Así, al elegir una determinada marca de omeprazol, el $44 \%$ afirmaba basar esta decisión en el precio, porcentaje que se redujo al $30 \%$ en el diclofenaco, al $26 \%$ en el ciprofloxacino y que no alcanzaba el $20 \%$ en el caso de enalapril, amoxicilina o paracetamol. En contraste, la afirmación de que el precio juega un papel importante en la elección de una marca comercial no se cumplía en el caso concreto de omeprazol y ciprofloxacino, donde eran más utilizadas las marcas más ca- ras y con menor variedad de presentaciones, incluso entre quienes afirmaban que el precio es una prioridad al prescribir.

La consideración de la utilidad terapéutica de un fármaco tampoco era un punto decisivo a la hora de recomendarlo. Así, substancias que eran consideradas de utilidad terapéutica baja por los facultativos eran prescritas hasta por un $70 \%$ de los mismos.

La prescripción inducida percibida suponía según los MAP el 39,7 \% (20,0) (IC $95 \%$ $[35,8 \sim 43,7])$ del total, y consideraban que el $15,9 \%(13,9)$ podría evitarse directamente. Entre las especialidades que más medicación inducida producían destaca Aparato cardiovascular (47 \%), seguida de Aparato locomotor $(12,5 \%)$ y Aparato respiratorio $(11,5 \%)$.

Respecto a la actitud del MAP frente a la medicación inducida, un $37,5 \%$ la sus- 
pendía sin esperar la opinión del especialista cuando creía que la indicación no estaba bien sustentada. Sin embargo, la escasa experiencia de uso (el $92 \%$ no sustituye) o el coste excesivo (el $75 \%$ no sustituye) no se manifestaban como determinantes a la hora de establecer un cambio de la medicación prescrita por otros niveles asistenciales.

Dentro del cuestionario se solicitaba a los facultativos que eligieran las tres medi- das que a su juicio eran más útiles para reducir el gasto farmacéutico (tabla 4). Considerando el porcentaje de médicos que eligió cada una de las posibilidades, la mayoría $(67,3 \%)\left(\mathrm{IC}_{95 \%}[57,3 \sim 75,9]\right)$ se decantó por el copago, seguido a distancia por la prescripción de genéricos $(39,4 \%)$ $\left(\mathrm{IC}_{95 \%} \quad[30,1 \sim 49,4]\right)$, la utilización de guías de prescripción $(38,4 \%) \quad\left(\mathrm{IC}_{95 \%}\right.$ $[29,1 \sim 48,4])$ y la implantación de precios de referencia $(25,0 \%) \quad\left(\mathrm{IC}_{95 \%}\right.$ $[17,2 \sim 34,6])$.

Tabla 4

Porcentaje de médicos que eligen cada una de las medidas propuestas para contener el gasto farmacéutico

\begin{tabular}{|lc|}
\hline & $\%(n)$ \\
\hline Copago & $67,3 \%(70)$ \\
Prescripción de genéricos & $39,4 \%(41)$ \\
Guías clínicas & $38,4 \%(40)$ \\
Precios de referencia & $25,0 \%(26)$ \\
Información de precios & $23,0 \%(24)$ \\
Sesiones clínicas & $18,2 \%(19)$ \\
Incentivos no monetarios & $12,5 \%(13)$ \\
Información personalizada & $12,5 \%(13)$ \\
Limitación de prestaciones & $11,5 \%(12)$ \\
Incentivos monetarios & $9,6 \%(10)$ \\
Asesoramiento farmacéutico & $8,6 \%(9)$ \\
Revisiones con colegas & $7,7 \%(8)$ \\
Autogestión & $6,7 \%(7)$ \\
Sanciones & $0,9 \%(1)$ \\
\hline
\end{tabular}

Entre los facultativos que elegían el copago el tiempo dedicado a la visita de los delegados farmacéuticos era significativamente menor (10,6 minutos frente a 16,0 minutos; $p<0,001)$, sin diferencias significativas en las restantes variables.
En la tabla 5 se resumen el porcentaje global de error de estimación de los facultativos y las principales asociaciones significativas halladas en el estudio. 
Tabla 5

Porcentaje global de error de estimación y principales asociaciones significativas

\begin{tabular}{|c|c|c|}
\hline \multicolumn{3}{|c|}{ Mayor número de delegados } \\
\hline Mayor número de TIS & $\rho=0,3$ & $\mathrm{p}=0,003$ \\
\hline
\end{tabular}

\begin{tabular}{|lcl|}
\hline & $\begin{array}{c}\text { Mayor porcentaje de error } \\
\text { en la estimación }\end{array}$ & \\
\hline Mayor número de pacientes/día & $\rho=0,28$ & $\mathrm{p}=0,03$ \\
Mayor número de delegados & $\rho=0,38$ & $\mathrm{p}=0,002$ \\
\hline
\end{tabular}

TIS: tarjetas sanitarias individuales $\rho$ coeficiente de correlación de Spearman.

\begin{tabular}{|lccc|}
\hline & $\begin{array}{c}\text { Partidarios del copago } \\
\text { como medida para redu- } \\
\text { cir gasto farmacéutico }\end{array}$ & $\begin{array}{c}\text { No partidarios del copa- } \\
\text { go como medida para re- } \\
\text { ducir gasto farmacéutico }\end{array}$ \\
\hline $\begin{array}{l}\text { Duración de la visita de los delegados farmacéuticos } \\
\text { (en minutos) }\end{array}$ & 10,6 & 16,0 & $\mathrm{p}<0,001$ \\
\hline
\end{tabular}

$\rho$ : coeficiente de Spearman.

\section{DISCUSIÓN}

Para interpretar adecuadamente los resultados obtenidos deben considerarse las conocidas limitaciones de los estudios transversales basados en cuestionarios ${ }^{9}$ y la representatividad de la muestra. La sospecha de intento de control de la prescripción por parte de la Administración pudo influir a la baja en el nivel de participación obtenido, aunque comparable a los alcanzados en estudios similares $^{8,10,11} \mathrm{y}$, asimismo, sesgar las respuestas en un sentido favorable a lo que se considera que debería ser un «buen profesional».

La única diferencia observada entre respondedores y no respondedores, respecto del tamaño de la población donde se ejerce, depende de una menor respuesta en los equipos de atención primaria de la capital, aunque creemos que esto no disminuye la representatividad de la muestra. Por otro lado, la cantidad limitada de datos obtenida puede no representar suficientemente las características subyacentes a un determinado comportamiento prescriptor.

El conocimiento que tienen los médicos del coste de los fármacos que prescriben es escaso, a juzgar por los resultados de este estudio, coincidentes con los de la mayor parte de la literatura consultada $a^{6-8,12-14}$.

Para evitar que el hecho de no prescribir un producto influyese en el desconocimiento de su precio se excluyeron del análisis aquellos casos en los que el facultativo afirmaba no prescribirlo. De cualquier forma, hay que considerar que elegir un determinado fármaco supone haberlo comparado con otros, y en esa comparación debería estar incluido el precio de las alternativas. Por otra parte, el método de selección de los fármacos se basó en la relación de fármacos más 
prescritos en la provincia con lo que, en general, se trataba de sustancias de muy amplio uso.

En la apreciación del coste de los medicamentos se comprueba un fenómeno de unificación de los precios, con sobrestimación de los más baratos y subestimación de los más caros. Este fenómeno ha sido observado de una u otra forma por la mayoría de los auto$\operatorname{res}^{5,7,8,12,13,15-17}$, siendo probablemente un reflejo del desconocimiento del precio del fármaco, que hace que se considere un valor similar para la mayoría de los productos.

Al igual que en otros estudios ${ }^{7,15,18,19}$ no se ha encontrado relación entre el grado de conocimiento del precio de los fármacos y las características sociodemográficas de los facultativos. Sin embargo, sí se observa asociación positiva entre error en la estimación y volumen de pacientes y de visitadores médicos. La presencia de estos últimos no condiciona un mayor conocimiento de los precios en contra de lo que parece lógico. De hecho, sólo un $1 \%$ de los facultativos considera el precio un aspecto de gran interés en la visita de los delegados farmacéuticos.

A pesar de que la mayoría de los médicos reconoce considerar el precio al prescribir, menos de la cuarta parte cree que deba ser una prioridad a la hora de seleccionar un fármaco. Otros autores ${ }^{10,12,14,19}$ encuentran a este respecto cifras similares, aunque al comparar con estudios realizados en otros países es preciso tener en cuenta las diferencias respecto a la financiación pública de los fármacos. Como es lógico, el hecho de que la mayor parte de la factura farmacéutica corra a cargo de la Seguridad Social hace que la consideración de la eficiencia de la prescripción esté menos presente en el prescriptor. En contraste, un estudio experimental ${ }^{20}$ ha demostrado que el conocimiento de los precios hace que la prescripción sea más económica.

Según los datos obtenidos, la IF ejerce una presión comercial directamente proporcional al «poder prescriptor» del facultativo medido en función de la población adscrita (como ya demostró Figueiras ${ }^{21}$ en un medio similar) y dedica una media de 60 horas anuales a ofertar información sobre fármacos $(7,5$ jornadas laborales de 8 horas al año, más de lo dedicado habitualmente a formación continuada). Esta atención a la información suministrada por la IF contrasta más todavía con el desconocimiento del precio que reflejan los datos. A este respecto, se constata con frecuencia que la publicidad ofrecida por la IF no lleva el precio del fármaco o es difícilmente legible $e^{12,16}$, salvo cuando la propia promoción introduce el precio como factor motivador. Por otro lado, un alto porcentaje de médicos (54\%) admite leer los informes/ensayos presentados por la IF (escasamente contrarrestados con datos de contenido crítico) y una amplia mayoría de los médicos cree adecuado el patrocinio de actividades formativas por parte de la IF (como señalan Caamaño et al $^{2}$, la IF es vista por los facultativos como una figura colaboradora frente a la Administración, de la que se destaca generalmente su papel fiscalizador). En nuestra encuesta, el $27 \%$ de los facultativos reconoce su participación, en todo caso legítima, en estudios promovidos por la IF, lo que facilita la creación de un cierto hábito prescriptor ya que la razón más importante para prescribir un fármaco es, probablemente, una buena experiencia con el producto que dé al médico una opinión personal favorable ${ }^{11}$. Así, un elevado porcentaje reconoce mantenerse fiel a una determinada marca comercial, generalmente a la de la compañía que desarrolla el fárma$\mathrm{co}^{18}$. Si, además, tal como se demostró en un estudio $^{22}$, un $47 \%$ de los médicos considera como fuente de actualización farmacológica a los visitadores médicos, parece necesario un serio esfuerzo por parte de gestores, colectivos y asociaciones médicas para fomentar una adecuada formación continuada en farmacología clínica.

Respecto al porcentaje de medicación que los facultativos perciben como inducida, en su más amplia acepción, éste coincide con las cifras encontradas en nuestro país, con valores que oscilan entre el 30 y el $50 \%{ }^{23-28}$, 
destacando el mayor volumen de medicación originada por los especialistas del área cardiovascular, dato coherente con la patología prevalente en nuestro medio y con los hallazgos de otros autores ${ }^{24,28}$.

Gallego $^{29}$, en Barcelona, encuentra prácticamente el mismo porcentaje de desacuerdo con la prescripción inducida. La elevada proporción de médicos que mantienen una medicación prescrita por otro a pesar de encontrarse en desacuerdo con la misma $(62,5 \%)$, lo que evidentemente genera malestar en el médico de atención primaria, debería ser motivo de reflexión en la Administración sanitaria para establecer mecanismos que lo impidan ${ }^{30}$.

Entre las alternativas para reducir el gasto farmacéutico destaca la apreciación mayoritaria de que el copago sería la fórmula más eficaz de conseguirlo. Figura a continuación la prescripción de genéricos, quizá como reflejo de su reciente introducción en el momento de la encuesta, y las actividades formativas, aunque si se consideran en su conjunto, las medidas de tipo puramente económico predominan sobre las formativas. Básicamente coinciden con lo encontrado por Alastrué et al ${ }^{15}$ en Valencia, si exceptuamos que en ese estudio no se ofrecía la alternativa del copago. No resulta fácil interpretar la apreciable diferencia existente entre el tiempo dedicado a la IF entre partidarios y no partidarios del copago como fórmula de control del gasto y creemos que merecería un estudio específico.

En opinión de los facultativos, la información sobre el gasto producido no parece ser de mucha utilidad como instrumento para la contención del gasto, dato coincidente con lo encontrado en estudios similares ${ }^{14,31}$. Otros métodos precisarían ser evaluados mediante estudios experimentales para probar su utilidad en este campo y, dado que los propios médicos así lo creen, es posible que una mayor formación ${ }^{32}$ en materias tales como la farmacoeconomía y algo tan simple como una adecuada información sobre el precio de lo que se receta ${ }^{33}$ pudiera ser de utilidad para conseguir una prescripción más racional, acciones que no deberían limitarse a los médicos de atención primaria, sino que, dado el elevado porcentaje de medicación inducida, convendría extender igualmente a los especialistas de segundo nivel ${ }^{34}$.

\section{AGRADECIMIENTOS}

Al Dr. Arturo Louro, Coordinador del EAP de Cambre (A Coruña) y a la Dra. María del Carmen Sánchez-Celaya, miembro del EAP Aldebarán (Madrid), por su colaboración en la realización de la prueba piloto del cuestionario.

\section{BIBLIOGRAFÍA}

1. Dirección General de Farmacia y Productos Sanitarios. Ministerio de Sanidad y Consumo. Indicadores de la prestación farmacéutica del Sistema Nacional de Salud a través de Receta. Año 2000. Inf Ter Sist Nac Salud 2001; 25 (2): 65

2. Caamaño F, Figueiras A, Gestal-Otero JJ. Condicionantes de la prescripción en atención primaria. Aten Primaria 2001; 27: 43-48.

3. Abanades JC, Cabedo V, Cunillera R, García JJ, Jolín L, Martín M et al. Factores que influyen en la prescripción farmacológica del médico de atención primaria. Aten Primaria 1998; 22: 391-398.

4. Figueiras A, Caamaño F, Gestal-Otero JJ. Incentivos de la industria farmacéutica a los médicos: problemas éticos, límites y alternativas. Gac Sanitaria 1997; 11: 297-300.

5. Oppenheim GL, Erickson SH, Ashworth C. The family physician's knowledge of the cost of prescribed drugs. J Fam Pract 1981; 12: 1027-1030.

6. Fowkes FGR. Doctors' knowledge of the costs of medical care. Medical Education 1985; 19 . 113-117.

7. Rowe J, MacVicar S. Doctors' knowledge of the cost of common medications. J Clin Hosp Pharmacy 1986; 11: 365-368.

8. Miller LG, Blum A. Physician awareness of prescription drug costs: A missing element of drug advertising and promotion. J Fam Pract 1993; 36: 33-36.

Rev Esp Salud Pública 2001, Vol. 75, N. ${ }^{\circ} 4$ 
9. Argimón JM, Jiménez J. Métodos de investigación. Barcelona: Ediciones Doyma SA; 1991.

10. Bradley CP. Factors wich influence the decision whether or not to prescribe: the dilemma facing general practitioners. Br J Gen Pract 1992; 42: 454-458.

11. Cialdella Ph, Figon G, Haugh MC, Boissel JP. Prescription intentions in relation to therapeutic information: a study of 117 french general practitioners. Soc Sci Med 1991; 33: 1263-1274.

12. Safavi KT, Hayward RA. Choosing between apples and apples: physicians' choices of prescription drugs that have similar side effects and efficacies. J Gen Intern Med 1992; 7: 32-37.

13. Mengual M, Guasch J, Falcó C, Segura J, Aguilar C. ¿Conocen el coste del tratamiento del asma los médicos de atención primaria? Aten Primaria 2000; 26: 295 .

14. Mengíbar FJ. ¿Existe conciencia de gasto farmacéutico en la atención primaria reformada? Aten Primaria 2001; 27: 408-411.

15. Alastrué JI, Meneu R, Peiró S. Análisis de las opiniones, actitudes y conocimientos de los médicos de los centros de salud de Valencia sobre la eficacia y la eficiencia en la prescripción de medicamentos. Aten Primaria 1998; 21: 165-171.

16. Glickman L, Bruce EA, Caro FG, Avorn J. Physicians' knowledge of drug costs for the elderly. J Am Geriatr Soc 1994; 42: 992-996.

17. Kolassa EM. Physicians' perceptions of prescription drug prices: their accuracy and effect on the prescribing decisión. Journal of Research in Pharmaceutical Economics 1995; 6(1): 23-37.

18. Taziaux P, Franck J, Ludovicy R, Albert A. A study of general practitioners' prescribing behaviour to the elderly in Wallonia, Belgium. Eur J Pub Health 1996; 6: 49-57.

19. Walzak D, Swindells 5, Bhardwaj A. Primary care physicians and the cost of drugs: A study of prescribing practices based on recognition and information sources. J Clin Pharmacol 1994; 34: 1159-1163.

20. Hart J, Salman H, Bergman M, Neuman V, Rudniki C, Gilenberg D et al. Do drug costs affect physicians' prescription decisions? J Intern Med 1997; 214: 415-420.

21. Figueiras A. Factores asociados a la prescripción de medicamentos: prescriptores y fuentes de información terapéutica. Un estudio farmacoepidemiológico [Tesis doctoral]. Santiago de Compostela: Universidad de Santiago de Compostela; 1993.
22. Nieto-Hernández T, Altimiras J, Buitrago F. Fiabilidad otorgada al Vademécum entre médicos de atención primaria. Aten Primaria 1996; 17: 247-250.

23. Franzi A, Placencia ML, Rodríguez L. Estudio de la prescripción inducida de las áreas básicas de salud de la Dirección de Atención Primaria Sabadell. Aten Primaria 1997; 20: 408-414.

24. Arroyo MP, Cano E, Ansorena R, Celay J, Cortés F, Estremera V. Prescripción delegada por especialistas en atención primaria. Aten Primaria 1995; 16: 538-544.

25. Lomeña JA, Cevallos M, Medina MT, Mediavilla E, Sarmiento F, Hernández JL. Gasto farmacéutico en atención primaria según el origen de las prescripciones. Aten Primaria 1996; 18: 35-38.

26. Pereiró I, Rodríguez R, Bartual MJ, Guijarro MD, Sánchez G, Suberviola V. Prescripción farmacológica en consultas de medicina general. Aten Primaria $1995 ; 15: 286-288$.

27. Gervás J. Sobre la prescripción inducida. Aten Primaria 1995; 15: 473-474.

28. Seguí M, Bartolozzi E, Ramos J, Llach A, Torrent M, Bresco E et al. Prescripciones de utilidad terapéutica baja inducidas en atención primaria. Aten Primaria 1998; 22: 227-232.

29. Gallego L, Fernández E, Flor X, Orfila F, Melió R Rodríguez M. Prescripción inducida: ¿estamos siempre de acuerdo? Aten Primaria 2000; 26: 308.

30. García JA, Cordón F. Prescripción inducida, ¿excusa o realidad? Aten Primaria 1998; 22: 619-621

31. Bloor K, Freemantle N. Lessons from international experience in controlling pharmaceutical expenditure II: influencing doctors. BMJ 1996; 312: 1525-1527.

32. Allery LA, Owen PA, Robling MR. Why genera practitioners and consultants change their clinical practice: a critical incident study. BMJ 1997; 314: $870-874$

33. Larizgoitia I, Rodríguez JR, Granados A y otros miembros del Comité Científico. Determinantes en la toma de decisiones clínicas en atención primaria: opinión de los profesionales. Aten Primaria 1998; 22: 505-513.

34. Gómez B, Trilla A, Vernet E, Corominas N, Codina $\mathrm{C}$, Ribas $\mathrm{J}$ et al. ¿Conocen los médicos el coste de los tratamientos y pruebas que solicitan? Rev Clin Esp 1996; 196: 523-528. 
ANEXO 1

CUESTIONARIO

1.- Edad:

3.- Años en ejercicio:

5.- Modelo: (Integrado, No integrado)

6.- Su centro está ubicado en una población de: (menos de 5.000 habitantes
2.- Sexo: (Mujer, Varón)

4.- Plaza: (Propietario, interino, Sustituto)

7.- Según corresponda, contestar una de las Siguientes (A o B):

A: N. ${ }^{\circ}$ aproximado de tarjetas sanitarias:

B: N. ${ }^{\circ}$ aproximado de cartillas:

8.- N. ${ }^{\circ}$ aproximado de pacientes/día (incluido recetas):

9.- ¿Recibe Ud. visitadores médicos?: (Sí, No, ns/nc)

10.- ¿Cuántos por día?:

11.- Tiempo promedio dedicado a la visita médica cada día (en minutos):

12.- Aspectos de la visita que más le interesan (marcar solo una): (Novedades, aspectos farmacológicos (cinética, interacciones, indicaciones...), precio, no le interesa nada, ns/nc)

13.- Habitualmente, prescribe las Novedades (marcar solo una): (Tan pronto como se las presentan, espera información escrita de otras fuentes, espera opinión de compañeros, espera la aceptación generalizada del fármaco, $\mathrm{ns} / \mathrm{nc}$ )

14.- ¿Lee habitualmente los artículos o ensayos clínicos que le presentan los laboratorios?

$\mathrm{NS} / \mathrm{NC}$

15.- ¿Suele participar Usted en ensayos/estudios promovidos por la industria farmacéutica? Sí
No
$\mathrm{Ns} / \mathrm{Nc}$

16.- ¿Cree adecuado que la industria farmacéutica patrocine cursos, seminarios, congresos...? Sí No $\mathrm{Ns} / \mathrm{Nc}$

17.- Habitualmente, ¿se fija Ud. en el precio de los medicamentos que le presentan? Sí

18.- ¿Cree Ud. que el médico debe considerar de una forma prioritaria el precio de los medicamentos que prescribe? Sí
No $\mathrm{Ns} / \mathrm{Nc}$

19.- De los siguientes fármacos, indique: una estimación de su precio, su utilidad terapéutica y si lo prescribe o no. (Por favor no consulte ni Vademécum ni ninguna otra fuente de información)

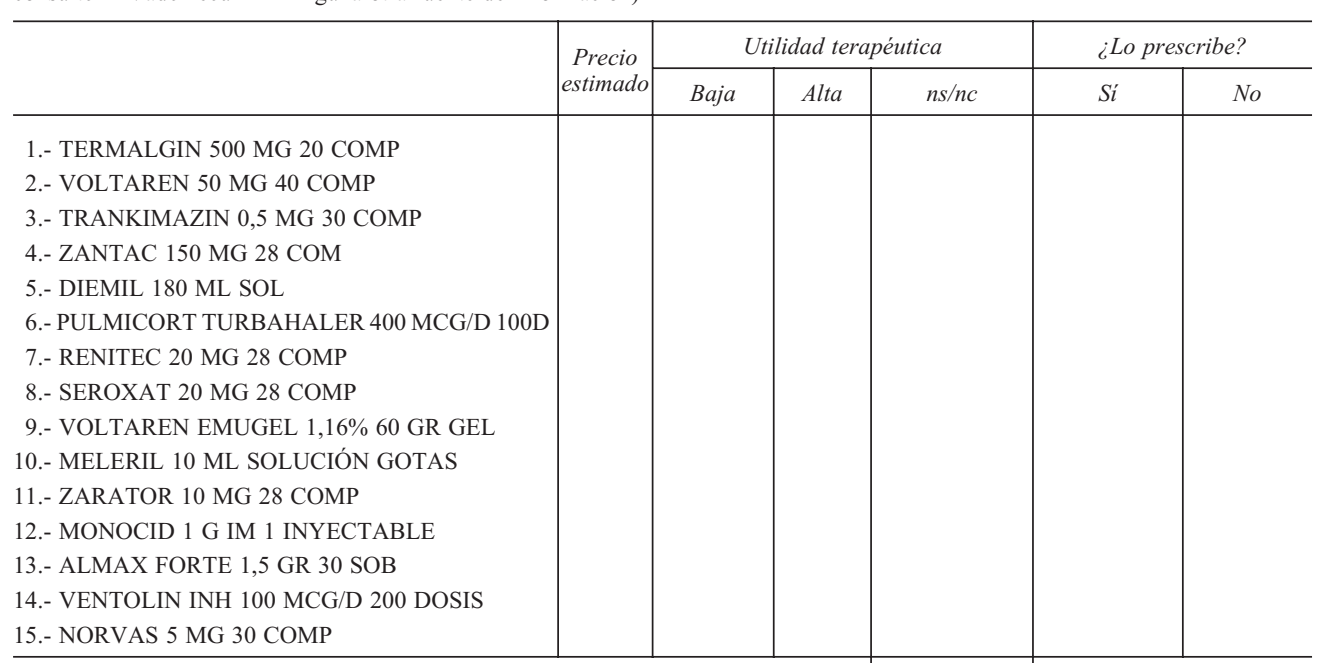


20.- De los siguientes principios activos, indique qué nombre comercial prescribe habitualmente y la razón más importante para hacerlo

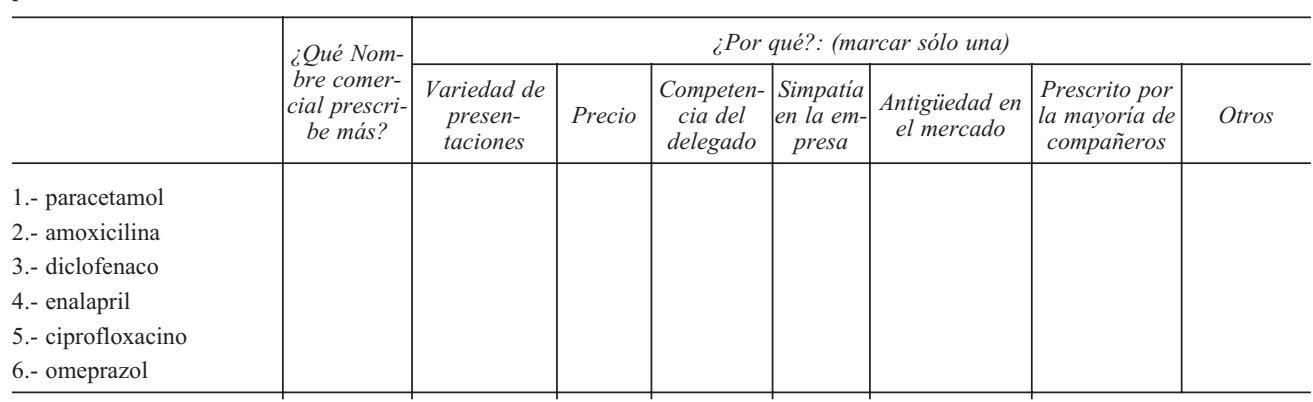

21.- ¿Suele ser fiel a las especialidades que prescribe?: (mucho, bastante, poco, ns/nc)

22.- ¿Qué porcentaje de sus recetas considera que es inducido por Atención Especializada?

23.- Del total de esa prescripción inducida, qué porcentaje considera usted como mal indicado, poco adecuado etc. y podría quizás evitarse?

24.- Señale por orden de importancia $\left(1 .^{\circ}, 2 .^{\circ}, 3^{\circ}\right)$, los tres grupos terapéuticos más inducidos por Atención Especializada: Aparato digestivo Terapia genitourinaria Aparato cardiovascular Terapia dermatológica

Aparato locomotor Terapia hormonal Antiobioticoterapia sistémica Aparato respiratorio Sistema nervioso Otorrinolaringología Oftalmología

Otros

25.- Respecto a la prescripción inducida, cuando usted considera que una medicación está poco indicada suele...: (...suspenderla directamente, ... esperar la opinión del especialista)

26.- Cuando una medicación inducida, aún correctamente indicada y pautada,

... es un fármaco nuevo con poca experiencia de uso, ¿suele usted sustituirlo por otro de uso más generalizado?: (Sí, No, ns/nc) ... es un fármaco con un coste, en su opinión, excesivo ¿suele usted sustituirlo por otro de menor coste?: (Sí, No, ns/nc) ... es un fármaco con una utilidad terapéutica, en su opinión, baja para esa indicación ¿suele usted...?: (suspenderlo, sustituirlo, mantenerlo, ns/nc)

27.- De las siguientes intervenciones, señale, en su opinión, las tres más importantes (solo tres), según su efectividad para reducir el gasto farmacéutico:

Guías de prescripción

Información periódica de precios

Comentarios/revisiones por colegas

Prescripción de genéricos

Información periódica personalizada del gasto

Incentivos No monetarios

Autogestión del Capítulo de Farmacia

\section{Sesiones clínicas}

Sanciones administrativas

Incentivos monetarios

Limitación de prescripciones cubiertas

Precios de referencia

Asesoramiento por un farmacéutico/farmacólogo

Copago (pensionistas y activos)

Muchas gracias por su colaboración 